\title{
Mating behavior of a Northern Italian population of Fusarium verticillioides associated with maize
}

Giovanni Venturini, Gemma Assante, Silvia L. Toffolatti \& Annamaria Vercesi

Journal of Applied Genetics

ISSN 1234-1983

Volume 52

Number 3

J Appl Genetics (2011)

52:367-370

DOI $10.1007 /$

s13353-011-0042-4

\section{Journal of Applied Genetics}

Approved by

The Polish Genetic Society

The Polish Society of Human Genetics

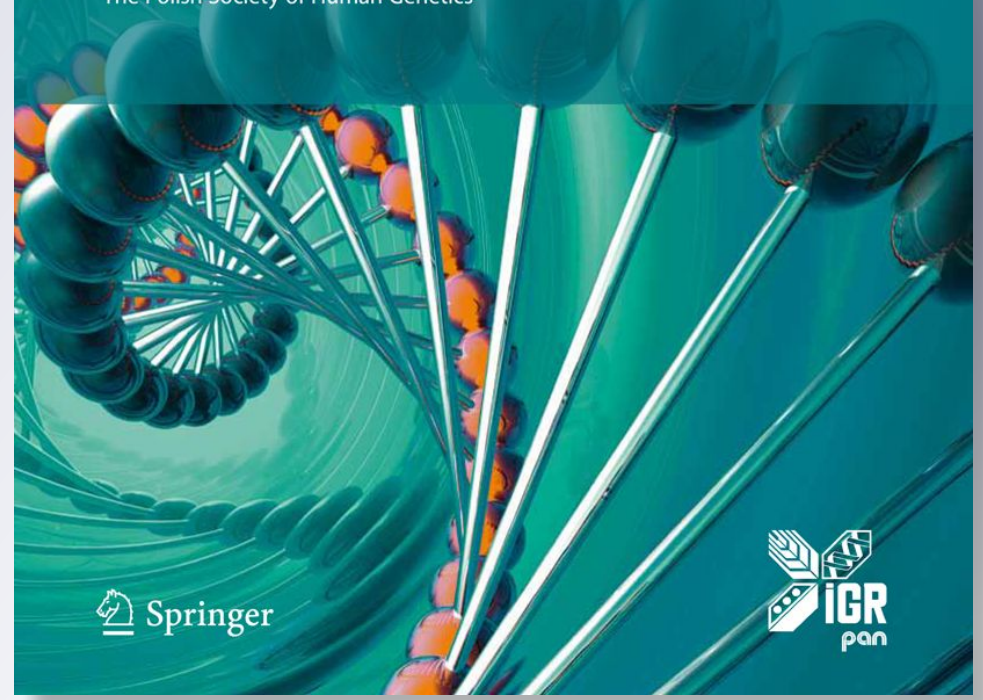

脰 Springer 
Your article is protected by copyright and all rights are held exclusively by Institute of Plant Genetics, Polish Academy of Sciences, Poznan. This e-offprint is for personal use only and shall not be self-archived in electronic repositories. If you wish to self-archive your work, please use the accepted author's version for posting to your own website or your institution's repository. You may further deposit the accepted author's version on a funder's repository at a funder's request, provided it is not made publicly available until 12 months after publication. 


\title{
Mating behavior of a Northern Italian population of Fusarium verticillioides associated with maize
}

\author{
Giovanni Venturini • Gemma Assante • \\ Silvia L. Toffolatti • Annamaria Vercesi
}

Received: 31 January 2011 /Revised: 4 March 2011 / Accepted: 15 March 2011 /Published online: 19 April 2011

(C) Institute of Plant Genetics, Polish Academy of Sciences, Poznan 2011

\begin{abstract}
Fusarium verticillioides, the most common causal organism of Fusarium stalk and ear rot of maize in Northern Italy, produces important mycotoxins such as fumonisins. Reproductive biology of $F$. verticillioides has been widely studied in numerous maize growing areas, but up to now no information is available on the mating behavior and genetic structure of this plant pathogen in Italy. Mating type and female fertility distribution and effective population number, $N_{e}$, were assessed for a population of $181 \mathrm{~F}$. verticillioides strains isolated from three fields located in Lombardia region (Northern Italy) during 2007-2008 maize growing season. The ratio of MAT1:MAT-2 was significantly different from the theoretical 1:1 ratio expected in an idealized population in which individuals mate at random. The frequency of hermaphroditic strains was $20 \%$ of the total population. $N_{e}$ for mating type was $89 \%$ of the count (total population) and the $N_{e}$ for male or hermaphrodite status was $55 \%$. The number of isolates that can function as the female parent limited $N_{e}$ in the examined population. Under equilibrium cycle, assuming that female fertility has been lost due to selection and mutation rate during asexual reproduction, sexual reproduction needed to occur only once per 40 to 118 asexual generations to maintain this level of sexual fertility.
\end{abstract}

Keywords Female fertility - Gibberella moniliformis . Mating type

G. Venturini $(\bowtie) \cdot$ G. Assante $\cdot$ S. L. Toffolatti $\cdot$ A. Vercesi Dipartimento di Produzione Vegetale sezione di Patologia Vegetale, Università degli Studi di Milano,

via G. Celoria 2,

20133 Milano, Italy

e-mail: giovanni.venturini@guest.unimi.it
Fusarium verticillioides (Sacc.) Nirenberg [synonym Fusarium moniliforme Sheldon, teleomorph Gibberella moniliformis Wineland synonym Gibberella fujikuroi (Sawada) Ito in Ito \& Kimura mating population A], a heterothallic fungus belonging to the Sordariomycetes Erikss. \& Winka, greatly affects maize production in temperate areas, such as Lombardia, the main corn producing region located in Northern Italy, and represents a potential threat to human and animal health due to its ability to produce fumonisins, a well-known group of harmful mycotoxins (Munkvold 2003).

Sexual reproduction in $F$. verticillioides requires the presence of two genetically distinct fungal isolates carrying alternate forms of mating types, referred to as mating types MAT-1 and MAT-2 (Kerényi et al. 1999). In addition, the male/female/hermaphroditic nature of the mating participants for successful sexual crosses is an important feature in F. verticillioides. The extent of sexual reproduction and its contribution to primary inoculum greatly influences both the evolutionary potential and epidemiology of numerous fungal plant pathogens. The frequencies of the two mating types and of male/hermaphrodite polymorphism in field population affect the amount of sexual crossing that can occur within a population. In fungal field populations, due to possible mutation or selection, the repeated asexual cycles reduce the frequency of hermaphrodites leading to a loss of female fertility. Reduction in female fertility can shift species to a mitosporic form which in turn has less adaptive advantages toward the selection pressure. The effect of genetic drift in the loss of female fertility during sexual reproduction can be estimated from the effective population number, $N_{e}$ (Leslie and Klein 1996). Moreover, $N_{e}$ is usually used to evaluate populations when mating is not randomly distributed and when individual members of the population do not contribute equally to the gene pool of 
the progeny (Caballero 1994). The mating behavior of $F$. verticillioides populations isolated mainly from cereals has been studied in several areas of the world (Chulze et al. 2000; Cumagun 2007; Danielsen et al. 1998; Leslie and Klein 1996; Mansuetus et al. 1997; Reynoso et al. 2006). To the best of our knowledge, no detailed information is currently available on the genetic structure of $F$. verticillioides populations in Italy as well as in other European maize growing areas, apart from the mating type assessment carried out by Moretti et al. (2004) on 24 Italian strains, isolated from maize plants showing ear and stalk rot. The objectives of this study were (1) to determine the mating types within a population of $F$. verticillioides isolated from maize in Lombardia, (2) to calculate the relative proportion of $F$. verticillioides female-sterile and hermaphroditic strains, and (3) to estimate the $N_{e}$ for the examined $F$. verticillioides population.

The present investigation was carried out on 181 strains of $F$. verticillioides, arbitrarily selected among the fungal population isolated during the 2007/2008 maize cropping season from three fields located in Lombardia (Venturini et al. 2011). Mating type idiomorphs of $F$. verticillioides strains were identified by sexual crossing procedures as described by Leslie and Summerell (2006). Sexual crosses were carried out on carrot agar with standard tester strains for G. moniliformis FGSC7600 (MAT-1) and FGSC7603 (MAT-2) as female parents (Fungal Genetics Stock Center, Kansas City, MO, USA) and field $F$. verticillioides isolates as male parents. A set-up with tester strains alone served as control. A cross was considered fertile when a cirrhus of ascospores oozing from a mature perithecium was observed 2-6 weeks after fertilization. Female fertility of the field isolates was determined in crosses in which the field isolates were used as the female parents and the standard testers as the male parents. All crosses were repeated twice. Difference in frequencies was tested for significance using the chi-square test with a single degree of freedom (PAST software ver. 1.95; Hammer et al. 2001). The equations proposed by Leslie and Klein (1996) were used in order to calculate the $N_{e}$, based on the mating type ratio $\left[N_{e(m t)}\right]$ and the relative frequency of female-sterile and hermaphrodite strains $\left[N_{e(f)}\right]$, and also the average number of asexual generations per sexual generation. $\left[N_{e(m t)}\right]$ was determined by the equation $N_{e(m t)}=\left(4 N_{\text {MAT-1 }} N_{\text {MAT-2 }-2) /(}\left(N_{\text {MAT-1 }}+N_{\text {MAT-2 }}\right)\right.$, where $N_{\text {MAT-1 }}$ was the number of $F$. verticillioides strains with MAT-1 idiomorph and $N_{M A T-2}$ was the number of strains carrying the other idiomorph MAT-2. This equation, first derived by Wright (1931) for diploids with two discrete sexes, is used to reduce population size of ascomycetes if both mating types are not equally frequent. $\left[N_{e(f)}\right]$ was calculated by the equation $N_{e(f)}=\left(4 N^{2} N_{h}\right) /\left(N+N_{h}\right)^{2}$, where $N$ was the total number of $F$. verticillioides strains and $N_{h}$ was the number of hermaphrodites. This equation is based on the observation that in field populations many isolates are fertile as males but not as females, due to the mutations and selection against hermaphrodites during vegetative propagation: if asexual reproduction is an important part in the life cycle of the fungus, female-sterile strains could dominate the population. Moreover, when such population reproduces sexually, the relative lack of hermaphrodites reduces the effective population size.

The average number of female sterile mutations per strains $(M)$ was calculated assuming a random distribution of the mutations and using the frequency of the hermaphrodites as the zero term in the Poisson distribution, $e^{-M}\left(M^{i} / i !\right)$ where $M$ was the mean number of mutations per strains, $i$ was the number of mutations in a given class of strains. The hermaphrodites were the only members in the class $i=0$.

MAT-1 and MAT-2, the mating type idiomorphs of the $F$. verticillioides population consisting of 181 strains isolated from maize in Northern Italy, segregated in a ratio 120:61 (Table 1). According to the chi-square test $(P<0.001)$, this

Table 1 Female fertility and inbreeding effective numbers for $F$. verticillioides populations

\begin{tabular}{|c|c|c|c|c|c|}
\hline \multirow[t]{2}{*}{ Mating ratio } & \multirow[t]{2}{*}{$N_{f s}^{\mathrm{a}}: N_{h}^{\mathrm{b}}$} & \multicolumn{2}{|c|}{ Effective population number, $N_{e}$} & \multirow[t]{2}{*}{ Origin } & \multirow[t]{2}{*}{ Reference } \\
\hline & & Mating type, $N_{e(m t)}{ }^{\mathrm{c}}$ & Male/hermaphrodite dimorphism, $N_{e(f)} \mathrm{c}$ & & \\
\hline $120: 61$ & $145: 36$ & 89 & 55 & Italy & this study \\
\hline $27: 23$ & $35: 15$ & 81 & 42 & Philippines & Cumagun (2007) \\
\hline 129:74 & $98: 105$ & 93 & 90 & Argentina & Reynoso et al. (2006) \\
\hline $23: 47$ & $62: 8$ & 88 & 37 & Argentina & Chulze et al. (2000) \\
\hline $16: 23$ & $10: 29$ & 97 & 98 & Costa Rica & Danielsen et al. (1998) \\
\hline $59: 17$ & $39: 37$ & 69 & 88 & Tanzania & Mansuetus et al. (1997) \\
\hline $237: 446$ & $342: 341$ & 91 & 89 & United States & Leslie and Klein (1996) \\
\hline
\end{tabular}

${ }^{a} N_{f s}$ is the number of female-sterile male-fertile isolates;

${ }^{b} N_{h}$ is the number of hermaphroditic isolates;

${ }^{c} N_{e(m t)}$ and $N_{e(f)}$ are expressed as a percentage of the actual count. 
ratio was significantly different from the theoretical 1:1 ratio expected in an idealized random mating population (Leslie and Klein 1996). Since MAT idiomorphs were not present at equal frequencies in the $F$. verticillioides population, asexual reproduction was likely to occur in the field isolates. However, the occurrence of the two opposite mating types in the $F$. verticillioides population implied that the population members were capable of sexual reproduction. Among the 181 strains examined, 36 isolates, accounting for the $20 \%$ of the total $F$. verticilloides population, were female-fertile (Table 1). Among the 36 hermaphrodites, MAT-1 and MAT-2 segregated in a 25:11 ratio. To determine the population size in relation to the size of population mating at random, the effective population number based on mating type ratios $\left[N_{e(m t)}\right]$ and on the relative frequency of female-sterile and hermaphrodites strains $\left[N_{e(f)}\right]$ was estimated (Table 1).

The mean number of female sterility mutations per strains $(M=1.6)$ showed a decrease in the number of hermaphrodites after each sexual reproduction cycle.

The mating type ratio (120:61), different from the theoretical 1:1 ratio, resulted in a decrease of the effective population number to $89 \%$ of the count (Table 1). The number of female-sterile strains (145 strains), representing $80 \%$ of the total $F$. verticillioides strains, caused a reduction of the effective population number in comparison with the population size expected if the entire population strains had been hermaphrodite, precisely $45 \%$ reduction of the count (Table 1).

The relative frequencies of sexual and asexual reproduction of $F$. verticillioides could be also inferred in this study from the assessment of the effective population number, $N_{e}$. $N_{e}$ is mainly affected by two factors: mating type and female sterility. In F. verticillioides, like in other Fusarium species belonging to the G. fujikuroi species complex, female fertility was far more important in determining $N_{e}$ than the relative proportions of strains belonging to different mating types (Leslie and Klein 1996). Even if in the Italian population MAT-1 frequency was twofold the MAT-2 frequency (120:61), $N_{e}$ was still near $90 \%$ of the total count.

On the other hand, female-sterile:hermaphrodite ratio equal to $145: 36$ led to a significant decrease of $N_{e}$ to $55 \%$ of the total count. Such decrease of $N_{e(f)}$ of the examined $F$. verticillioides population indicated the dominant role of asexual recombination, selection and drift rather than the sexual recombination in determining the genetic variation. Leslie and Klein (1996) argued for the absence of sexual reproduction in local populations of G. fujikuroi species complex by the presence of female sterility mutations resulting in an increased vegetative propagation capability.

If the population was at equilibrium, data could also be used to estimate the range in which the percentage of hermaphrodites could fluctuate and the relative number of asexual generations per sexual generation (Leslie and Klein 1996). Depending on the assumptions concerning the combined effects of mutation rate on female sterility and the selection against hermaphrodites during the asexual portion of the life cycle, the average number of asexual cycles per sexual cycle, varied from 40 to 118 in the examined population, and the frequency of hermaphrodites in such an equilibrium population could range from 10 to $45 \%$ (observed value, $20 \%$ ). The number of female sterility mutations per strains obtained by applying the model of Leslie and Klein (1996) to the F. verticillioides population $(M=1.6)$, was higher than those calculated by Leslie and Klein (1996); Mansuetus et al. (1997) $(M=0.7)$ and Reynoso et al. (2006) $(M=0.6)$. The $F$. verticillioides population associated with maize grown in Northern Italy therefore seemed to be characterized by a high tolerance to female sterility mutations, during asexual reproduction, at which female fertility was lost. The comparison between the $N_{e(f)}$ values obtained in the present study with those calculated by other Authors pointed out that the structure of the examined Italian population more closely resembles the relatively infertile Argentinean (Chulze et al. 2000) and Philippine populations (Cumagun 2007). Agricultural practices, such as tillage, under which maize was grown in the examined fields, were not likely to provide opportunity for sexual recombination, as already pointed out by Reynoso et al. (2006). Moreover, a wider sampling region should allow to isolate $F$. verticillioides strains which could show a major level of female fertility (Reynoso et al. 2006). This is the first contribution on mating behavior of $F$. verticillioides population in Italy and results suggest the need to further investigate by genotyping studies other Italian $F$. verticillioides populations in order to clarify the pattern of genetic variation within and between populations.

Acknowledgments The Authors thank G. Russo for his help in data analysis.

\section{References}

Caballero A (1994) Developments in the prediction of effective population size. Heredity 73:657-679

Chulze SN, Ramirez ML, Torres AT, Leslie JF (2000) Genetic variation in Fusarium section Liseola from no-till maize in Argentina. Appl Environ Microbiol 66:5312-5315

Cumagun CJR (2007) Female fertility and mating type distribution in a Philippine population of Fusarium verticillioides. J Appl Genet 48:123-126

Danielsen S, Meyer UM, Funck Jensen D (1998) Genetic characteristics of Fusarium verticillioides isolates from maize in Costa Rica. Plant Pathol 47:615-622

Hammer $\varnothing$, Harper DAT, Ryan PD (2001) PAST: paleontological statistics software package for education and data analysis. Paleontol Electron 4:1-9 
Kerényi Z, Zeller KA, Hornok L, Leslie JF (1999) Molecular standardization of mating type terminology in the Gibberella fujikuroi species complex. Appl Environ Microbiol 65:40714076

Leslie JF, Klein KK (1996) Female fertility and mating type effects on effective population size and evolution in filamentous fungi. Genetics 144:557-567

Leslie JF, Summerell BA (2006) The Fusarium laboratory manual. Blackwell Publishing, Oxford

Mansuetus ASB, Odvody GN, Frederiksen RA, Leslie JF (1997) Biological species in the Gibberella fujikuroi species complex (Fusarium section Liseola) recovered from sorghum in Tanzania. Mycol Res 101:815-820
Moretti A, Mulè G, Susca A, Gonzàlez-Jaén MT, Logrieco A (2004) Toxin profile, fertility and AFLP analysis of Fusarium verticillioides from banana fruits. Eur J Plant Pathol 110:601-609

Munkvold GP (2003) Epidemiology of Fusarium diseases and their mycotoxins in maize ears. Eur J Plant Pathol 109:705-713

Reynoso MM, Torres AT, Chulze SN (2006) Biological species in the Gibberella fujikuroi species complex isolated from maize kernels in Argentina. Plant Pathol J 5:350-355

Venturini G, Assante G, Vercesi A (2011). Fusarium verticillioides contamination patterns in Northern Italian maize during the growing season. Phytopathol Mediterr (in press)

Wright S (1931) Evolution in Mendelian populations. Genetics 143: 175-189 